\title{
Riboflavin assisted corneal collagen cross linking in patients with progressive Keratoconus: effect on visual and morphological changes
}

\author{
Anusuya Bhattacharyya ${ }^{1}$, Phulen Sarma ${ }^{2}$, Kalyan Das ${ }^{1}$ \\ ${ }^{1}$ Ophthalmology, Sri Sankaradeva Nethralaya, Guwahati Assam, India, ${ }^{2}$ Pharmacology PGIMER Chandigarh, India
}

Introduction In progressive Keratoconus patients, ultraviolet A light is used to induce covalent cross-link bonds between corneal collagen to strengthen it. Riboflavin has photosensitizing property. In this study we want to evaluate the efficacy and safety of riboflavin assisted collagen cross linking in north eastern Indian population.

Materials and methods A prospective observational study was designed with 60 eyes of progressive keratoconus 30 cases, 30 control were enrolled in the study. Data was collected at baseline, 1, 3 and 6 months following the riboflavin assisted collagen cross linking. Primary outcome was change in Kmax value. Secondary outcomes were Kmin, uncorrected visual acuity UCVA, best corrected visual acuity BCVA, contrast sensitivity, refractive parameters cylindrical power, spherical equivalent and spherical power and cell morphology parameters endothelial cell count and percentage hexagonality. SPSS version 20 was used for data analysis. Study was started after obtaining instiutional ethical committee permiossion.

Result The Case group showed significant decrease in $\mathrm{K}$ max and Kmin from baseline. No such change was seen in the control group. SimK showed significant improvement in the treatment group compared to the control at 6 months. Improvement in UCVA and BCVA was seen in the treatment group at 3 and 6 months, but in the control group it deteriorated. Treatment resulted in significant improvement in contrast sensitivity and the result was significantly different between the two groups at 6 months. The refractive improvements cylindrical power, spherical equivalent and spherical power were statistically significant at the end of 6 months. No significant difference was noted between the two groups in terms of endothelial cell count and percentage hexagonality.

Conclusion Use of topical riboflavin assisted collagen cross linking resulted in significant improvement of visual aquity, contrast sensitivity, refractive outcomes and SimK value at the end of 6 months in patients with progressive Keratoconus, which suggest improvement. 